\title{
Effects of Airflow Deflection Angle in Diffuser on Forced Response Caused by Impeller-diffuser Interaction in Centrifugal Compressors
}

\author{
Sun Peng ${ }^{1,2, a}$, Wang Yanrong ${ }^{1,2}$ and Zhang Xiaobo ${ }^{1,2}$ \\ ${ }^{1}$ School of Energy and Power Engineering, Beihang University, China \\ ${ }^{2}$ Collaborative Innovation Center for Advanced Aero-Engine, China
}

\begin{abstract}
Aeroelasticity has always been a significant issue of compressors. Impeller-diffuser interaction (IDI) produces periodic aerodynamic excitation which forces the blades to vibrate in centrifugal compressors, and eventually causes the damage of blades. Therefore, it is of great importance to investigate the IDI in centrifugal compressors and the effects on the vibration of blades. In this paper, it is discussed in detail that the deflection angle of the airflow in diffuser affects the IDI of the centrifugal compressor and the vibration of blades. The results show that if deflection angle is positive, the interaction of the diffuser on impeller and the vibration of main blades are decreased. However, on condition that the deflection angle is excessive, the interaction of the diffuser on the flow field of impeller will be intensified, and the vibration of the main blades will be stronger. Therefore, it is necessary to reasonably control the range of the deflection angle so that centrifugal compressors are able to work in a safer environment.
\end{abstract}

\section{Introduction}

Compared with the parameters of axial compressors, the rotating speed of centrifugal compressors is much higher and the blades are slighter, which results in a much larger stress of blades. If blades vibrate due to aeroelastic problems during the operation of compressor, especially when resonance occurs, fatigue cracks may appear on blades, resulting in high-cycle fatigue (HCF) problems and the damage of blades. Therefore, it is necessary to investigate the factors that are likely to lead to aeroelastic problems, so as to avoid the failure of blades and compressors.

Forced response of a centrifugal compressor caused by IDI is one of the main problems of aeroelasticity. IDI in a centrifugal compressor can be defined as a group of mechanisms which cause the differences in flow phenomena and performance of the two components between isolated and coupled operation, including unsteady and other effects [1].

As for the research of IDI, Koth [2] compared the effects of vaned diffuser and vaneless diffuser on impeller, and found that steady simulations of flow field were approximately the same, but unsteady excitation was detected obviously under the case of the vaned diffuser. Lusardi [3] compared three different centrifugal compressors and concluded that unsteady periodic pressure occurs at the leading edge of diffuser, which perturbs the upstream flow field along the surface of blades. And the wave may have a phase difference between the pressure surfaces and suction surfaces of blades, which

\footnotetext{
${ }^{a}$ Corresponding author : sunpeng1994@buaa.edu.cn
} 
can amplify the perturbation. Lusardi [3] and Borm [4] proved that some general softwares for CFD calculations (such as CFX, Fine Turbo, etc.) are able to well simulate the unsteady aerodynamic loads generated by IDI in centrifugal compressors and results are obtained accurately.

A number of researchers have worked on figuring out the parameters that affect the IDI of centrifugal compressors. Gould [5] proposed three main parameters that affect unsteady aerodynamic loads of the IDI: radial clearance, DeHaller number, and Mach number. The smaller radial clearance is set, the stronger the IDI is. Zemp [6] proved that radial clearance has a greater effect on the vibration of the blades under higher mass flow conditions compared to lower mass flow conditions. In addition, according to the results of experiments, Skoch [7] found that the diffusion ability of diffuser is greatly affected by the airflow deflection angle in the diffuser. However, for the vaned diffusers whose vanes are NACA-shaped, which are used more widely in centrifugal compressors, there is still a lack of relevant research. Besides, there are few studies focus on the relationship between the deflection angle and the vibration of impeller blades caused by IDI.

In order to figure out the effects of deflection angle of the airflow in diffuser on forced response of the impeller blades caused by IDI, a detailed study is conducted, including the simulation of flow field in centrifugal compressor, modal analysis and transient analysis of main blades of impeller. In the simulation, the deflection angle of the airflow in diffuser is determined by changing the camber line of the diffuser vanes, and then the purpose of changing the deflection angle of airflow in the diffuser is achieved.

\section{Controlling equations}

In terms of the simulations of the flow field of compressor, NUMECA software is used to solve the three-dimensional unsteady Reynolds Average Navier-Stokes (RANS) equations. Spalart-Allmaras model is used as turbulence model, and the Phase Lagged model is adopted on the interface of impeller and diffuser, which can make sure that the all computational domains are reduced to a single domain without changing the number of blades or vanes and considerably shorten the time for calculation [8]. Gerolymos [9] and Zante [10] have shown that accurate results can be obtained by the Phase Lagged model in the flow field calculation of a single-stage compressor, including a stage of stator and a stage of rotor. In terms of the structure of blades, the finite element method is used. ANSYS software is used to solve the three-dimensional structural dynamic equation:

$$
\boldsymbol{M} \ddot{x}(t)+\boldsymbol{C} \dot{x}(t)+\boldsymbol{K} x(t)=\boldsymbol{F}(t)
$$

where $\boldsymbol{M}, \boldsymbol{C}, \boldsymbol{K}$, and $\boldsymbol{F}$ represent mass matrix, damping matrix, stiffness matrix and force matrix respectively.

\section{Introductions of model}

A scaled model of the DDA 404-III centrifugal compressor impeller is used as the model in this paper, which contains 15 main blades and 15 splitter blades. The vaned diffuser contains 24 NACA-shaped vanes. It has been proved that the weight of centrifugal compressor is lighter with NACA-shaped vanes compared with wedge-shaped vanes, but the phenomenon of IDI is more severe, so it is more worth to investigate centrifugal compressor with NACA-shaped vanes. The model of centrifugal compressor is shown in Figure 1.

The deflection angle of airflow in diffuser is defined as

$$
D A=\alpha-\beta
$$

where $\alpha$ and $\beta$ are the angles between the camber line of a vane and radial lines, as shown in Figure 2. $r_{1}, r_{2}$ and $r_{3}$ are the radii of the trailing edges of blades, the leading edges of vanes and the trailing edges of vanes. A series of diffuser airflow deflection angles are set in order to figure out the effects 
of deflection angle on the strength of IDI, the area affected by IDI and the vibration stress of blades. Four diffusers with different deflection angles are selected as cases for analysis, including deflection angles are $0^{\circ}, 5^{\circ}, 14^{\circ}$ and $29^{\circ}$, as shown in Figure 3.

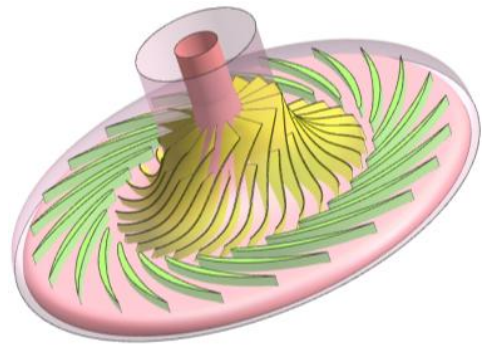

Figure 1. The model of centrifugal compressor

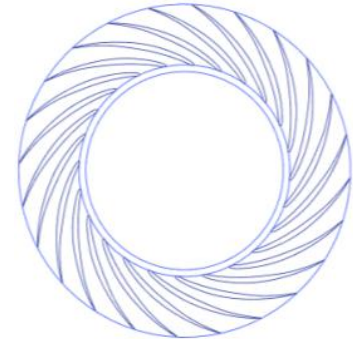

(a) $\mathrm{DA}=0^{\circ}$

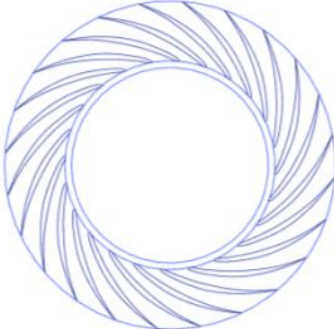

(b) $\mathrm{DA}=5^{\circ}$

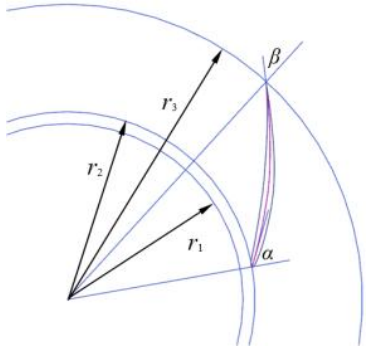

Figure 2. The definition of $\alpha$ and $\beta$

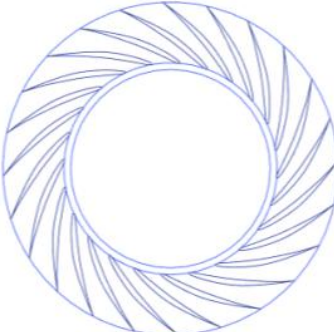

(c) $\mathrm{DA}=14^{\circ}$

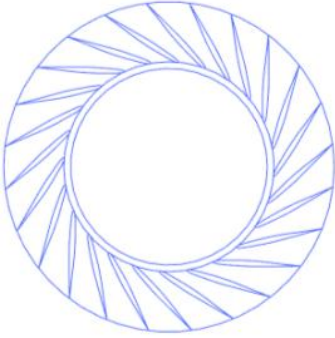

(d) $\mathrm{DA}=29^{\circ}$

Figure 3. Four cases with different deflection angles in diffuser

The boundary conditions of flow field are set, the inlet of which is $101.3 \mathrm{kPa}$ of total pressure and $288.15 \mathrm{~K}$ of total temperature. Outlet total pressure has been set as $350 \mathrm{kPa}$, making the efficiency of centrifugal compressor reaches the maximum. The grids of flow field are generated by Autogrid automatically, the total number of which is 1.8 million. The quality check is passed. In all cases, the total number of grids is kept close to avoid the difference of results caused by the density and quality of the grids. The tip clearance of the blades is set as a typical value, while the tip clearance of the vanes is set as 0 .

\section{The effects of deflection angle on flow field unsteadiness caused by IDI}

Steady and unsteady simulations of flow field are carried out for all cases. The passing frequency can be derived with equation (3):

$$
f_{\mathrm{p}}=\frac{n N_{\mathrm{s}}}{60}
$$

where $n$ is rotating speed, $N_{\mathrm{s}}$ is the number of vanes, which is 24 in this paper.

In order to figure out the law of flow field unsteadiness caused by IDI with different deflection angles, a series of monitoring points are selected, which located at $70 \%$ chord, $85 \%$ chord and trailing edge of main blades. The pressure changing with time at monitoring points are extracted, and the components of pressure at monitoring points are obtained after filtering and spectrum analysis. The results of spectrum analysis are shown in Figure 4. 


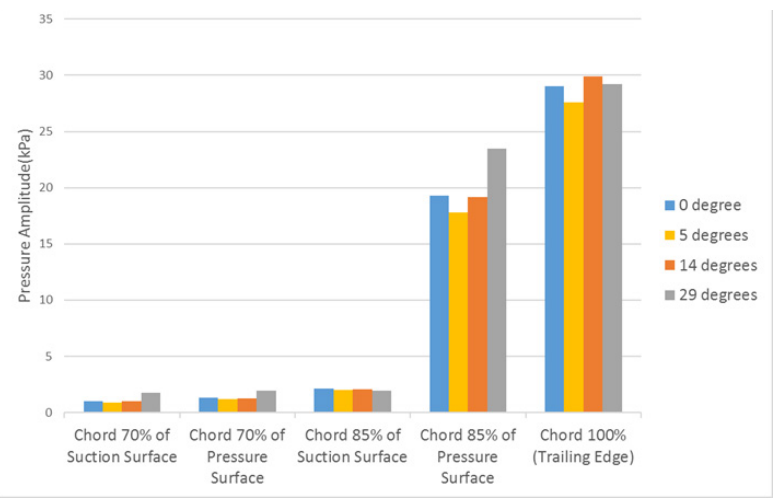

Figure 4. The results of spectrum analysis

According to the result, it can be concluded that:

1 IDI plays an important part in the flow field near the trailing edge of the blade. At the trailing edge, the pressure fluctuation amplitude is about $30 \mathrm{kPa}$. By contrast, the value of pressure in average at the trailing edge is $245 \mathrm{kPa}$, of which the amplitude reaches almost $1 / 8$.

2 There is a big difference in the area of the flow field affected by IDI between suction surface and pressure surface of the main blade. The amplitude in the pressure surface at chord $85 \%$ of main blade is almost $20 \mathrm{kPa}$, while it is reduced to less than $3 \mathrm{kPa}$ in the suction surface. Therefore, the effects of IDI on the pressure surface is wider than that on the suction surface. However, the area is still relatively limited. The flow field from the leading edge of the blade to chord $70 \%$ is hardly affected by IDI.

3 With the increase of the deflection angle, the pressure fluctuation amplitude at the $85 \%$ chord length of the pressure surface decreases at first and then increases, reaching the minimum when deflection angle equals to $5^{\circ}$. But when the airflow deflection angle is no more than $14^{\circ}$, the pressure fluctuation amplitude does not change much, but it is obviously smaller than the amplitude in the case where deflection angle is excessive. It can be confirmed that when the deflection angle is not quite large, there is almost no significant change in the strength of the IDI of a specific point and the area of flow field affected by the IDI. However, if the deflection angle is excessive, the pressure fluctuation amplitude and the area of flow field affected by IDI will both increase.

\section{Model analysis of main blade}

Previous studies have shown that the problems of vibration caused by aeroelasticity happened to mean blades more frequently. Therefore, the main blade is selected as the model for analysis. The finite element model of the main blade has been established, as shown in Figure 5. The titanium alloy TC4 has been selected as the material of the blade, the key parameters of which are shown in Table 1. The temperature field has not been taken into account. Model analysis of main blade has been carried out, according to the result of which Campbell diagram of main blade is plotted, as shown in Figure 6 . The points where the stress amplitude is respectively large are selected as monitoring points for transient analysis carried out subsequently. There are 5 points are selected, as shown in Table 2. The distributions of the equivalent stress (SEQV) of the $6^{\text {th }}$ mode and the $7^{\text {th }}$ mode are shown in Figure 7 , in which the position of the monitoring points are marked at the same time.

Table 1. Material Parameters of TC4

\begin{tabular}{|c|c|c|c|}
\hline Material & Density $\left(\mathbf{k g} / \mathbf{m}^{\mathbf{3}}\right)$ & $\begin{array}{c}\text { Elastic } \\
\text { Modulus(GPa) }\end{array}$ & Poisson's Ratio \\
\hline TC4 & 4440 & 109.1 & 0.34 \\
\hline
\end{tabular}




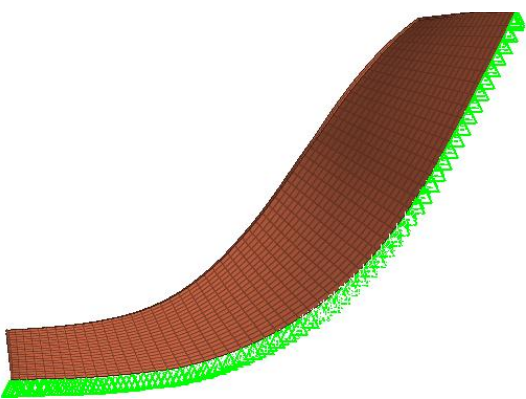

Figure 5. Finite element model of main blade

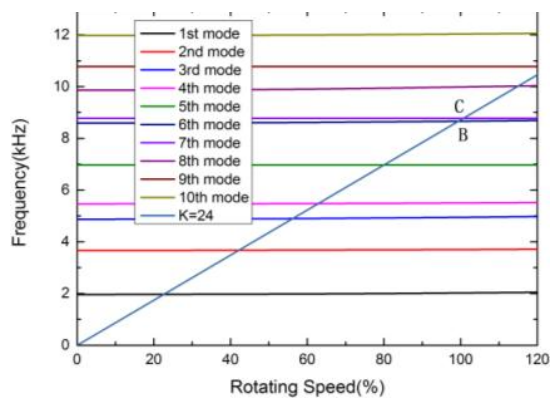

Figure 6. The Campbell diagram of main blade

Table 2. Selected points and working points

\begin{tabular}{|c|c|c|c|c|}
\hline Mode & $\begin{array}{c}\text { Point that } \\
\text { intersects with } \\
\text { line K=24 }\end{array}$ & $\begin{array}{c}\text { First principal } \\
\text { stress }\end{array}$ & $\begin{array}{c}\text { First principal } \\
\text { stress }\end{array}$ & $\begin{array}{c}\text { von Mises } \\
\text { stress }\end{array}$ \\
\hline $6^{\text {th }}$ Mode & B & No.1137 & No.8 & No.1137 \\
\hline $7^{\text {th }}$ Mode & C & No.1136 & No.195 & No.1126 \\
\hline
\end{tabular}

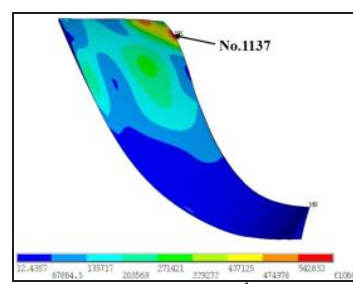

(a) SS of the $6^{\text {th }}$ mode

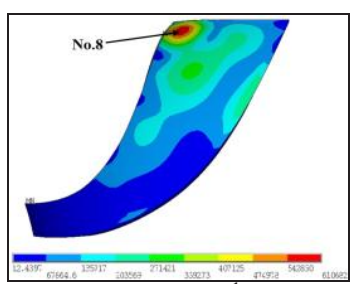

(b) PS of the $6^{\text {th }}$ mode

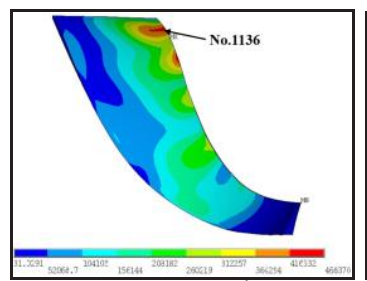

(c) $\mathrm{SS}$ of the $7^{\text {th }}$ mode

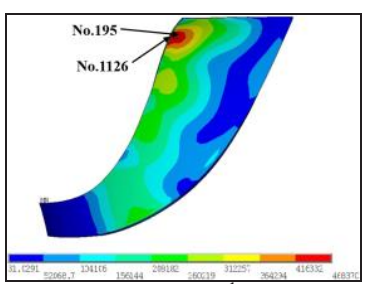

(d) PS of the $7^{\text {th }}$ mode

Figure 7. The distribution of SEQV of main blade

\section{The effects of deflection angle on the vibration amplitude of blades}

The transient analysis results of monitoring points are extracted, and frequency components are derived by spectrum analysis with Fourier Transform method. The high-frequency components are omitted for the amplitudes are quite small. Only the amplitudes of top three low-frequency components are extracted, and the results are connected by smooth curves, as shown in Figure 8, where $1 \mathrm{x}, 2 \mathrm{x}$, and $3 \mathrm{x}$ are used to represent the component of passing frequency, double of passing frequency, and triple of passing frequency respectively.

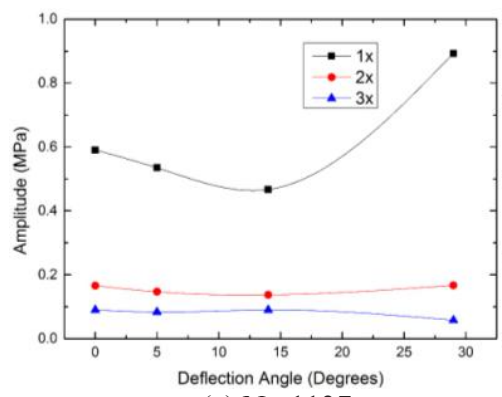

(a) No.1137

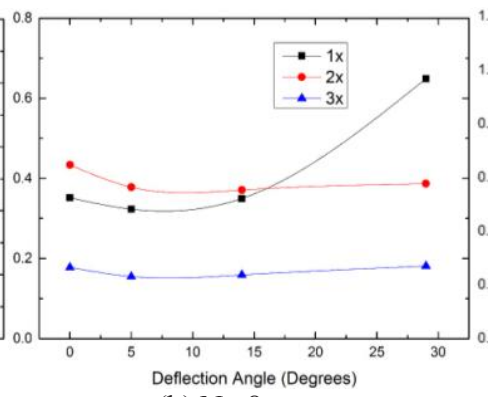

(b) No.8

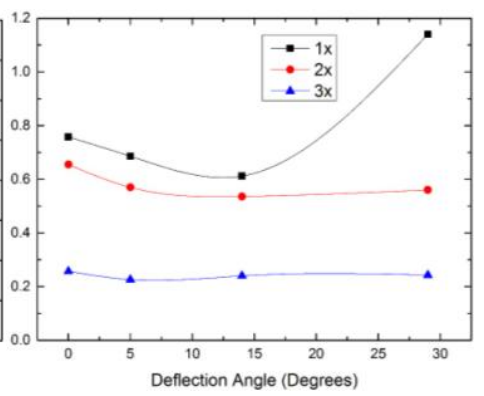

(c) No.1136 


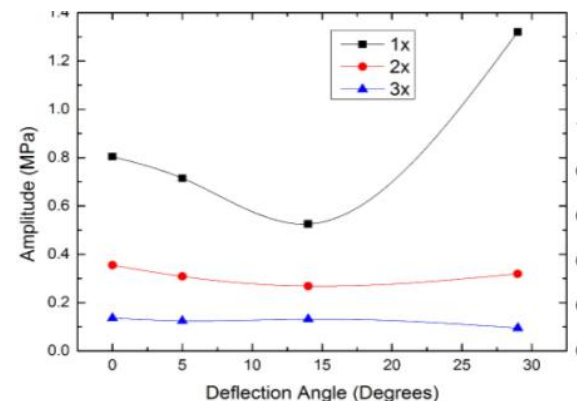

(d) No.195

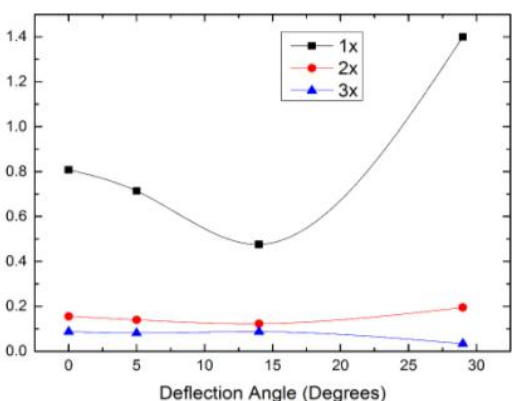

(e) No. 1126

Figure 8. Stress amplitudes of monitoring points

The results of all monitoring points show that the stress amplitude of $1 \mathrm{x}$ is greatly affected by deflection angle, but the stress amplitudes of $2 x$ and $3 x$ are hardly affected by deflection angle. With the increase of deflection angle, the stress amplitude of $1 \mathrm{x}$ has a tendency of decreasing at first and increasing subsequently. The value of the stress amplitude reaches the minimum when deflection angle is between $5^{\circ}$ and $15^{\circ}$. In addition, the stress amplitude of $1 \mathrm{x}$ is much larger when the deflection angle is $29^{\circ}$, the value that actually exceeds the reference value. In other words, main blades are more likely to fail attributed to high vibration stress on condition that deflection angle is excessive in centrifugal compressor. Therefore, it should be fully taken into account that the deflection angle should not be designed to be excessive in the design of the diffuser, so that the probability of failure of main blades caused by massive stress can be reduced.

\section{Conclusions}

According to the simulation of flow field of a centrifugal compressor and transient analysis of blades, the effects of deflection angle of the airflow in diffuser on the vibration of main blades caused by IDI is clarified. The following conclusions are deduced from computed results:

1 IDI plays an important part in the flow field near the trailing edge of the blade, and the affect of IDI on pressure surface is greater than that on suction surface. On condition that deflection angle is excessive, pressure fluctuation amplitude increases considerably.

2 With the increase of the deflection angle, main blade stress amplitude of $1 \mathrm{x}$ has a tendency of decreasing slowly at first and increasing subsequently. The value of the stress amplitude reaches the minimum when deflection angle is between $5^{\circ}$ and $15^{\circ}$ in the centrifugal compressor introduced in this paper. While stress amplitudes of $2 \mathrm{x}$ and $3 \mathrm{x}$ are hardly affected by the deflection angle. Therefore, the deflection angle should not be designed to be excessive.

\section{References}

1. U.Z. Kai, H.E. Gallus, R. Niehuis, J. Tur. 125, 545 (2003)

2. H. Koth, K. Oeder, J. E. Gas Tur. Pow. 688 (1987)

3. C. Lusardi, MIT thesis (2012)

4. O. Borm, H.P. Kau, ASME conf. (2012)

5. K. Gould, ASME conf. (2007)

6. A. Zemp, R.S. Abhari, J. Tur. 135, 1507(2012)

7. G.J. Skoch, J. Tur. 127, 107 (2004)

8. L. He, J. Tur. 112, 714 (1990)

9. G.A. Gerolymos, G.J. Michon, J. Neubauer, J. Prop. Pow. 18, 1139 (2012)

10. D.V. Zante, J. Chen, M. Hathaway, J. Tur. 130, 1067 (2008) 